\title{
Peripheral facial palsy associated with Epstein-Barr virus infection in a pediatric patient
}

Marianna Immacolata Petrosino, Giovanni Prezioso, Marina Cerruto, Nadia Rossi, Francesco Chiarelli

Department of Pediatrics, “G. D’Annunzio” University, Chieti, Italy

Submitted: 17 July 2016

Accepted: 30 July 2016

Arch Med Sci Civil Dis 2016; 1: e63-e65

DOI: 10.5114/amscd.2016.61787

Copyright @ 2016 Termedia \& Banach

\author{
Corresponding author: \\ Giovanni Prezioso \\ Department of Pediatrics \\ "G. D’Annunzio" University \\ Via dei Vestini 5 \\ 66100 Chieti, Italy \\ Phone: +390871358690 \\ E-mail: gprezioso@hotmail.it
}

A 13-year-old girl was admitted to the pediatric ward for acute onset of mouth deviation to the left, drooping of the right corner of the mouth, poor right eyelid closure, right hypoacusis, and reduced taste perception. She also complained of right ear pain a week ago, and she had low-grade fever 2 weeks ago.

Her history showed only respiratory allergy.

At first evaluation she was afebrile and she also showed inability to wrinkle the right side of the forehead, right lagophthalmos, loss of the right nasolabial fold and inability to puff her right cheek and to lift the ipsilateral mouth corner. Otoscopy revealed hyperemic tympanic membranes bilaterally, more evident to the right. Enlarged lateral cervical lymph nodes, painless and tender to palpation were found bilaterally. The remaining physical findings and neurologic examination were normal. Hence peripheral facial paralysis was diagnosed.

Laboratory examinations revealed a mild increase in white blood cell count with neutrophilia (leukocytes: $17.03 \times 10^{9} / \mu$; neutrophils: $82.7 \%$ ). C-reactive protein was negative. Serologic tests for Varicella Zoster virus, Epstein-Barr virus (EBV), Paramyxovirus and ToRCH (Toxoplasma gondii, Rubella, Cytomegalovirus and Herpes Simplex virus) were performed. The EBV IgM titer was found positive $(49.9 \mathrm{U} / \mathrm{ml}$, reference range: $0-20 \mathrm{U} /$ $\mathrm{ml})$, with positive $\mathrm{lgG}(139 \mathrm{U} / \mathrm{ml}$, reference range: $0-20 \mathrm{U} / \mathrm{ml})$. The polymerase chain reaction (PCR) result for EBV DNA was positive, so facial paralysis associated with EBV infection was diagnosed. Magnetic resonance of the brain was normal and no abnormalities of the infratemporal tract of the facial nerve were detected.

Thus, oral acyclovir (800 mg 5 times a day) plus oral steroid therapy with prednisone ( $2 \mathrm{mg} / \mathrm{kg} /$ day) were administered for 10 days with slow and gradual improvement of symptoms and subsequent prednisone tapering.

After discharge, the girl underwent a weekly follow-up until nearly complete remission of right facial muscle weakness, after less than 2 months.

Acute peripheral facial paralysis is the most common acute mono-neuropathy [1], and it is often a challenge for clinicians. Its incidence has been reported as 21.1 per 100,000 per year for children < 15 years old [2].

The causes of pediatric facial nerve paralysis (Table I) can be congenital or acquired, but the idiopathic form or Bell's palsy is the most frequent (40-75\% of cases) [3].

Acquired facial paralysis can often be caused by viral infections. In the present case, although the clinical and laboratory findings did not show a typical EBV infection pattern (neutrophilia with no symptoms or signs 
Table I. Causes of pediatric facial nerve paralysis (modified from Ciorba et al. [3])

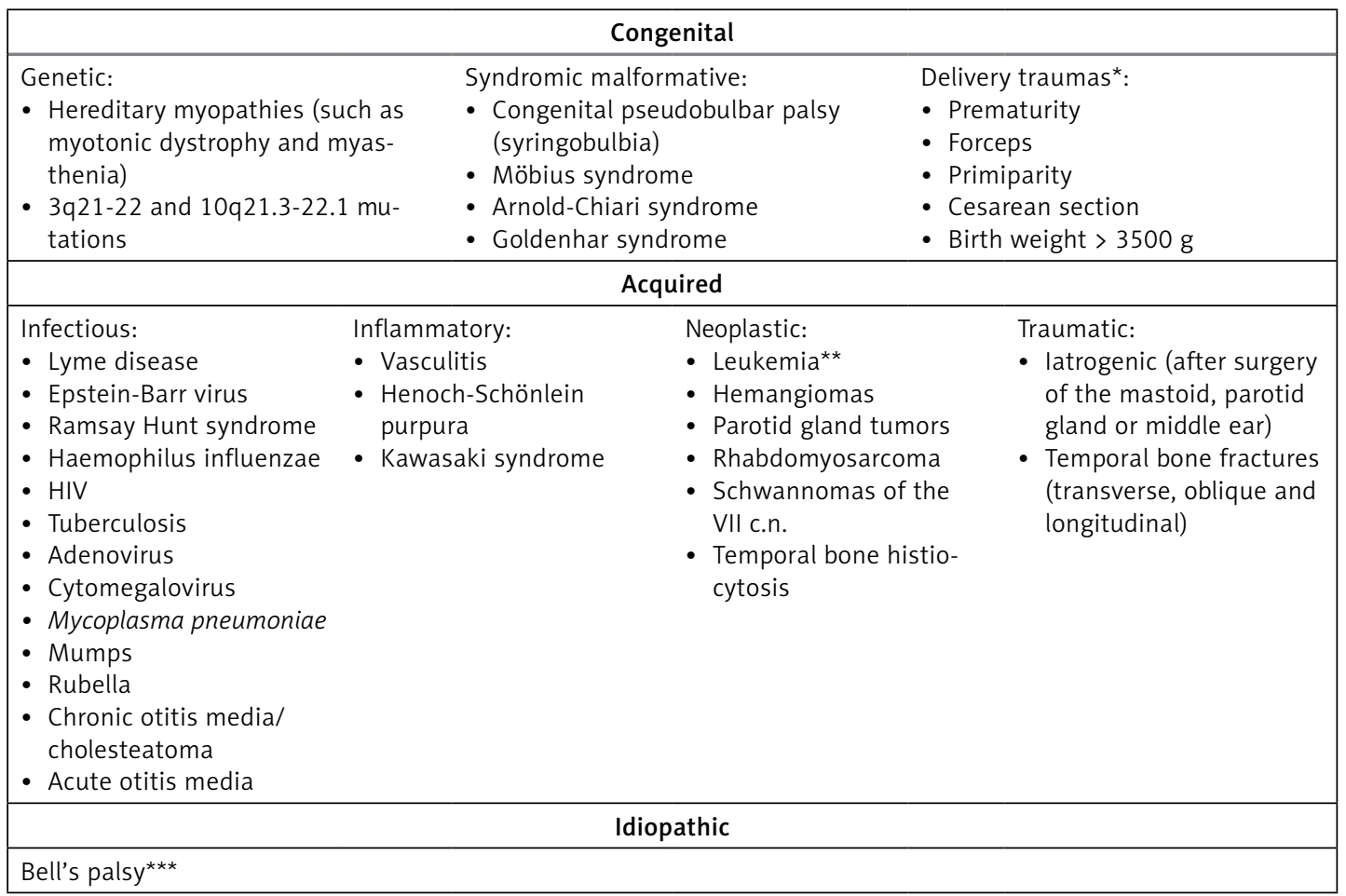

*Prognosis: These cases usually have a favorable prognosis, with infants recovering the full functionality of the seventh cranial nerve within a few months without sequelae. ${ }^{* *}$ Pediatric facial nerve paralysis associated with leukemia is bilateral in many cases. ${ }^{* * *}$ Prognosis: About $70 \%$ of Bell's palsy cases have a favorable prognosis with spontaneous resolution within 3 months, without sequelae.

of infectious mononucleosis), an EBV etiology was strongly suspected on the basis of serological findings, their temporal relationship with paralysis onset and the negative history for different acquired or congenital causes.

Neurological complications, such as cranial nerve palsies, Guillain-Barrè syndrome, meningoencephalitis, and mononeuritis multiplex, occur in $1-5 \%$ of all patients with acute EBV infection [4]. Neurological manifestations may be the only clinical signs, particularly in children, as in our case, leading to a delay in diagnosis. To our knowledge, only some cases of facial nerve paralysis associated with EBV infection are reported in children.

Approximately $40 \%$ of the cases of facial nerve paralysis associated with EBV are bilateral $[5,6]$.

Facial nerve paralysis can be complete or partial [7], peripheral or central. Peripheral paralysis is characterized by motor, sensory and visceral deficits of the hemi-face involved [3]. In addition to facial asymmetry at the examination of the face, impairment of facial autonomic innervation may be reported by affected patients: noise intolerance, ear pain, altered sense of taste, as in our patient, and decreased tearing [8].

Paralysis severity at disease onset can influence the degree of recovery: a severe paralysis hardly achieves a total recovery of nerve function [3]. According to the House-Brackmann scale, a clinical indicator of severity and progress of the disease, grades I and II usually have good outcomes, grades III and IV are characterized by moderate residual dysfunctions and grades $\mathrm{V}$ and $\mathrm{VI}$ are negative predictors $[3,8]$. So, even if usually self-limited, the facial paralysis can lead to important consequences. In about $5 \%$ of cases, paralysis can show residual sequelae such as synkinesis, contractures, facial spasms [3], corneal abrasion, autonomic dysfunction (decreased tearing) and psychosocial effects of facial deformity [8].

A careful history is important for timely diagnosis, considering that the most important cause of peripheral facial paralysis is idiopathic, which remains a diagnosis of exclusion [9].

It is necessary to examine the time course and the onset of the facial paralysis (a progressive onset, > 3 weeks, can suggest a neoplastic cause), the external auditory canal, the mastoid region, the eardrum, all the associated symptoms and comorbidities [3].

A blood count should be carried out when children are affected. Serologic tests should be performed when the history is suggestive for specific etiology. Our case shows that serology against EBV is essential in the diagnostic management of children with facial paralysis. Imaging tests are not routinely recommended [3]. The majority of children recover within 6 months [8], like our patient; however, the younger the patient, the better the prognosis [8]. 
The management of facial paralysis is related to its severity and etiology, if a specific cause is identified.

Oral corticosteroids in children within 3 days of symptoms onset are recommended, preferably prednisone $1-2 \mathrm{mg} / \mathrm{kg}$ per day for 10 days, followed by gradual dose reduction [3]. In the case of permanent paralysis, surgical techniques associated with rehabilitative approaches are required [3].

This case report provides an important clinicopathological correlation in pediatric age, because, as previously reported in the literature, it draws attention to EBV infection as a potential cause of acute facial paralysis in children without a history of other acquired or congenital causes. It also provides significant descriptions of this disease with potential important sequelae that can help clinicians in diagnosis. Furthermore, we provide another experience in the use of corticosteroids in children with acute facial paralysis, supporting the usefulness of these drugs. However, further studies are necessary to determine the effectiveness of corticosteroids in facial paralysis. It may also be useful in the future to estimate the incidence of the various pathogenic agents causing acute facial paralysis in children.

\section{Conflict of interest}

The authors declare no conflict of interest.

\section{References}

1. Baugh RF, Basura GJ, Ishii LE, et al. Clinical practice guideline: Bell's palsy. Otolaryngol Head Neck Surg 2013; 149: S1-27.

2. Jenke AC, Stoek LM, Zilbauer M, Wirth S, Borusiak P. Facial palsy: etiology, outcome and management in children. Eur J Paediatr Neurol 2011; 15: 209-13.

3. Ciorba A, Corazzi V, Conz V, Bianchini C, Aimoni C. Facial nerve paralysis in children. World I Clin Cases 2015; 3: 973-9.

4. Portegies P, Corssmit N. Epstein-Barr virus and the nervous system. Curr Opin Neurol 2000; 13: 301-4.

5. Terada K, Niizuma T, Kosaka Y, Inoue M, Ogita S, Kataoka N. Bilateral facial nerve palsy associated with Epstein-Barr virus infection with a review of the literature. Scand I Infect Dis 2004; 36: 75-7.

6. Diedler J, Rieger S, Koch A, Parthé-Peterhans S, Schwaninger M. Bilateral facial palsy: Epstein-Barr virus, not Lyme disease. Eur J Neurol 2006; 13: 1029-30.

7. de Araujo MR, Azenha MR, Capelari MM, Marzola C. Management of Bell's palsy: a report of 2 cases. J Can Dent Assoc 2008; 74: 823-7.

8. Biebl A, Lechner E, Hroncek K, et al. Facial nerve paralysis in children: is it as benign as supposed? Pediatr Neurol 2013; 49: 178-81.

9. Kaygusuz I, Gödekmerdan A, Keleş E, et al. The role of viruses in idiopathic peripheral facial palsy and cellular immune response. Am J Otolaryngol 2004; 25: 401-6. 